\title{
On the Creative Design of Amphibious Vehicles in a 3D Virtual Classroom Using OpenSim
}

\author{
Hung-Cheng Chen
}

\begin{abstract}
In this study, we have demonstrated a possible way to inspire students without engineering background to design amphibious vehicles of the future world. Our virtual classroom is facilitated with an open-source 3D world simulator, i.e., OpenSim, on a private computing cloud. We adopted the concept of the creative thinking spiral to help the students design their amphibious cars or boats following the principles of bionics engineering and fluid dynamics. In the stages of imagine and creation, students have to carefully balance the contributions from various aspects including the shape of the vehicles, the hydrodynamic forces on the water and on the land and the power consumption for amphibious maneuvering. Besides that, due to their limit skills of 3D modeling, we encourage the students to explain their design philosophies in details on a Facebook group. The peer comments received from the participants may lead to fresh idea of design and can result constructive reflections in the next cycle of the creative thinking spiral.
\end{abstract}

Index Terms-Amphibious vehicle, virtual reality, creative thinking spiral.

\section{INTRODUCTION}

In recent decades, the effect of climate change has drawn much attention about global warming effect of the Earth. This effect leads to tremendous disasters all over the world. The major consequences of this effect result from the sea level rises by the over-melting of the ice sheets in Antarctica and Greenland for instance. Meanwhile, the global warming effect also strengthens the intensity of the tropical cyclones that result to heavy rains, flood and mud flow over the urban or rural areas. On March 11, 2011, the Tohoku earthquake triggered tremendous tsunami waves that reached heights of up to 40.5 meters and, in the Sendai area, travelled up to 10 $\mathrm{km}$ inland. This supernatural disaster is terrible and is very difficult to escape by ordinary vehicles.

This study is motivated by asking that if it is possible to design imaginative amphibious vehicles by college students without engineering background in a general education course on sustainability and environment. This is because we are interested in inspiring students from interdisciplinary backgrounds to design amphibious vehicles with the knowledge of global warming effect and its associate disasters For this simulation purpose, we have built a $3 \mathrm{D}$ virtual

Manuscript received December 30, 2015; revised June 24, 2016. This work was supported in part by the National Science Council of Taiwan under contract No's NSC 100-2511-S-412-002-MY2. We are grateful to the National Center for High-performance Computing for computer time and facilities.

Hung-Cheng Chen is with the Faculty of Center of General Education, Yu Da University of Science and Technology, Miao-Li, Taiwan (e-mail: hungcheng@gmail.com). classroom by an open-source 3D world builder, i.e., the OpenSimulator, to help the students being immersive and learned in the virtual world.

In order to benefit from the interdisciplinary arrangement of the class, we encouraged students to demonstrate their designs on a Facebook group whereas the peer comments are welcome to each other. Through the social interactions on the Facebook, the participants could have the chance to explain their ide amore comprehensively about how their vehicles can conquer the challenges from the "world under the sea" environments.

A detailed literature review is given on Section II. For the educational purpose, we adopted the philosophy of creative thinking spiral to enhance the design process of the vehicles in Section III. The learning and creation processes are also demonstrated in the 3D virtual world and Facebook group. In Section IV, we will give a brief discussion of this study and a concluding remark is presented in Section V.

\section{LITERATURE REVIEW}

\section{A. Building a 3D Virtual World for Education}

The applications of 3D virtual world platform are widely explored in recent years. The most famous one is the Second Life (SL) that is highly developed and is a paid service for all participants. The user interface and the function of SL are similar to those of the massively multiplayer online game (MMOG). The users of SL can customize the appearance of avatars and interact to each other via instant messaging, voice chat and gestures. The difference between SL and other MMOGs is the features that users in SL do not have predestinated goal or game play. The scenes in SL are totally been constructed and developed by the users. This salient feature of SL leads to the possibilities of creating a whole imaginative virtual world by the SL players. The SL also provides a programming language called Linden Script that enables the players to determine the rule of interaction in the world of SL.

Another SL-compatible, open-source 3D virtual world builder, i.e., Opensimulator (OpenSim), has drawn much attention in recent years, especially for the academic users [1]. [2] has built an OpenSim package named Universal Campus which provides a comprehensive virtual campus includes presentation room, conference room and lecture hall. In these places, the users can communicate to each by local chat, voice conferencing, as well as powerpoint-enable presentations. [3] has presented an underwater virtual aquarium called Fish4Knowledgeproviding a content-rich, interactive and educational place for users to navigate with. There are virtual 
fishes swimming around the underwater aquarium and it also displays a looped marine life video that was captured in the costal sea off South Taiwan. In the review article [4], Hew and Cheung concluded that the 3D virtual worlds such as Second Life or OpenSim can fulfill the following needs for the educational purposes such as 1) communication in a verbal or nonverbal way, 2) simulation as in a virtual campus or classroom, and 3) experiencing in a virtual landscape such as the mathematical Menger sponge structure constructed by [5].

\section{B. The Creative Thinking Spiral}

In [6], Professor Resnick proposed a conceptual learning model that is synthesized from the kindergarten approach of learning called the creative thinking spiral. This model is characterized by a spiraling cycle of Imagine, Create, Play, Share, Reflect, and back to Imagine and enables an efficient way of improving the creative-thinking skills. By observing the learning process in the kindergarten, he found that there exists a core process that evolves recursively as the children learning. This process includes a spiraling process in which the children imagine what they want to do, create a project following their ideas, play with their creations, and share their ideas and results with others. Finally, these steps will reflect on their experiences and eventually lead them to other fresh ideas or to create new projects. In this study, we will adopt this model into our 3D virtual classroom and try to seek for more creative designs of the amphibious vehicles.

\section{Developments of Amphibious and Bionic Vehicles}

The amphibious vehicles have a long history since the $18^{\text {th }}$ century [7]. In the early stage of development, most of them were designed from the concept of fabricating a ship with wheels. This design thinking is straightforward and it has been applied in many cases such as the Alligator boats used in the forest industry or the amphibious assault vehicle (AAV) used in the Marine Corps, for instance. A major difficulty of the design of boat-type amphibious vehicle is its travel speed on land is relatively slow than it is on the water. This is because the aerodynamics form drag on land is severe for those boat-type vehicles but its counter hydrodynamic drag is relatively small on the water. This contradiction is very crucial for the design and it plays a key role on the energy consumption issue.

On the other hand, the design thinking of fabricating a car with one or several propellers to an amphibious car is good for seeking a high speed on land by its streamlined shape with low aerodynamic drag. However, the ordinary shape of cars will create a huge amount of wave drag and its associate buoyance force is relatively small than that of the boat-type vehicle. In order to conquer the high wave drag for a amphibious car, the hydrofoil has been applied to produce an additional lift force and to decrease the wave drag. On the combination of the hydrofoils and the propeller, the amphibious car with hydrofoil can reach a high speed between 80 to $90 \mathrm{~km} / \mathrm{h}$ in [8].

On the other hand, designers of modern vehicle also adopt the idea of bionic engineering in order to seek for an optimal design effectively. In 2005, Mercedes-Benz's new bionic car whose sloping nose and trim silhouette were modeled directly after the small tropical boxfish [9]. During the design stage, they seek for examples in nature whose shape and structure can satisfy the requirement of an aerodynamic-efficient and structure-lightweight car. In their study, the optimal aerodynamic parameters calculated from the boxfish and a new lightweight construction framework learned from nature lead to a boxfish car for excellent performance and fuel consumption [10]. Recently, a "bionically inspired" vehicle made with a light-weighted, weatherproof fabric skin stretched over a 3D-printed skeletal frame was demonstrated in Geneva Motor Show 2015 [11]. In this study, students are encouraged to adopt the idea of bionic design both in the aspects of aerodynamics and structure.

\section{Design OF AmphiBious Vehicles}

In this section, we present the design results of the amphibious vehicles following the principle of the creative thinking spiral in our 3D virtual world. The results contain a detailed description on how to apply the principle of creative thinking spiral to design virtual amphibious vehicles in our classroom. In order to inspire the creativity of students, we will discuss some teaching strategies of how to conduct more students to participate in the learning process and to improve their designs through recursive social interactions.

In order to provide a powerful CPU and sufficient network bandwidth for the calculation by OpenSim, we fabricated our OpenSim virtual world on a cloud-based high-performance computer named Formosa 3 that is built by 76 nodes with 48GB memory per node. The Formosa 3 provides a cloud-based service byIaaS (Infrastructure as a Service)that can allocate various computing nodes for different purposes. In this study, we have applied a service up to four nodes that can satisfy the computing for about thirty concurrent users.

In this study, the course was delivered over ten hours in five weeks of which two hours per week. The design of the course is followed by the idea of the five-stage creative thinking spiral that will be explained in the following sections. There are fifteen students attending this course. Three of them have the background of 3D modeling and the other students have the backgrounds of literature, management and information technology.

\section{A. The Stage of Imagine}

Because the students has no prior knowledge of amphibious vehicles, we asked them to search via Google with several key words such as amphibious car or amphibious boat in the classroom in order to dig into the various designs by themselves. Most of them got focused on the shapes and functionalities of the vehicles. Meanwhile, we explained the fundamental ideas behind the design of the amphibious vehicles such as reducing the hydrodynamic wave drag of boats or enhancing the aerodynamic drag reduction of automobiles.

On the other hand, we also showed several homemade amphibious vehicles that were occasionally invented in natural disasters. During the 2011 monsoon season in Thailand, severe flooding occurred and spread through the provinces of northern, northeastern, and central Thailand along the Mekong and Chao Phraya river basins. Some people have made their homemade vehicles to escape from these floods and many of their stories have been uploaded on the 
YouTube such as in [12] for a floating bike or as in [13] for a floating motorbike.

At last, we also showed some interesting designs of vehicles that implement the principle of bionic design such as the boxfish car made by Mercedes-Benz. In this stage, we emphasized the concept of bionics and show that how this principle can be beneficial for the optimal engineering design.

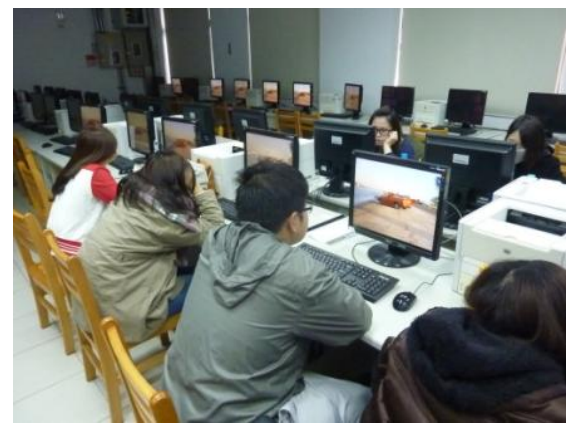

Fig. 1. Students are watching the video of amphibious vehicles

\section{B. The Stage of Creation}

In this stage, students began to learn how to build things in the virtual world. They learned some basic skills about how to use "prim" of which stands for primitive shape to construct objects, and to change their colors, shape or size, for instance. In this stage, the teacher and the teaching assistants advised the students for the 3D techniques of construction. Some of the students have the background of 3D modeling. They always asked for help to each other about how to create things as they intend to make. In this study, we do not teach the students with the Linden script of activating the object to move or to act interactively. On the other hand, we have pre-activated the required scripts on those objects that were necessary for the course. For instance, a "driving" script that enables the avatars to drive on the vehicle is essential for every participant.

In Fig. 2, we demonstrated the results of the selected designs by students. In Fig. 2(a), this vehicle is simply a traditional wooden boat that attaches six wheels and a small house on the boat. The design is easy to understand and it takes the wooden materials as the source of buoyance force. Apparently, the source of driving force in this vehicle may be the same as the traditional boat, that is, by pushing on the oars that were not shown in his design. In Fig. 2(b), a bionic vehicle looks like a duct that is shown that the boat intends to mimic the motion of a real amphibious duct. Interestingly, this boat has three wheels, one is under the head and the other two is under the body in its rear, for the purpose of driving on the land. On the other hand, there is a propeller mounted on the rear of the boat (which is not shown in Fig. 2(b)) and it provides the propelling force of the boat on the water. In order to protect the driver, there is an intensifying transparent cover on the boat.

For the bionic purpose, the duck beak is designed not only for the shape similarity but also for reducing the hydrodynamic wave drag on the water with the help of its bottom hull. On the other hand, there are two manipulative small wings fabricating along two sides, respectively. The design of the side wings can stabilize the vehicle and provide an elaborate attitude control of the vehicle on the water.
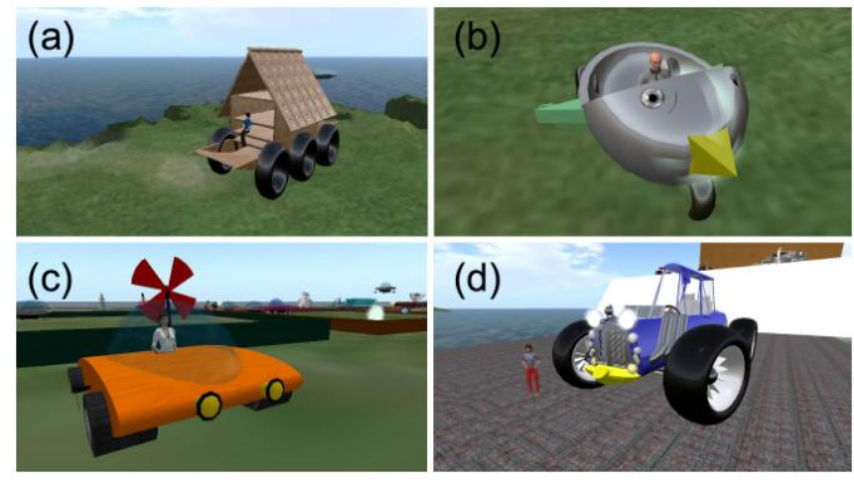

Fig. 2. Amphibious vehicles designed by students.

In Fig. 2(c), a wind-driven vehicle is built as an amphibious car. A small wind turbine mounted on the rear of the car is used for the purpose of power generation. The electricity generated by the wind turbine can provide the power of the vehicle. The shape of the vehicle is streamlined along the longitudinal cross-section that reduced the aerodynamic and a hydrodynamic form drag and provides an additional lift force on the water. On the other hand, the vehicle is wide in the latitudinal direction that provides sufficient buoyance force that is necessary for the vehicle on the water. The design of this streamline body may be originated from the idea of the hydrofoil that leads to a high-speed performance of the vehicle. Comparing with the case in Fig. 2(b), the wave-breaking resistance providing by the beak and bottom hull is compromised in this case in Fig. 2(c).

Finally, in Fig. 2(d), a classic car shows an elaborate design by a student who is capable of modeling 3D object in a beautiful way. In his design, he illustrated an appearance that is similar to that in the animated movie PorcoRossomade by the director Hayao Miyazaki. In this design, the arms of wheels provide a water-flapping mechanism that is similar to it is on the traditional steamship in the $18^{\text {th }}$ century. To avoid the overflow of the water, the exhaust pipe of the car is bended upward to the sky. Besides that, the bottom of the car is designed as similar as a ship hull in order to provide the wave breaking resistance on the water. Under a sufficient power of the engine, it can provide a powerful thrust with high rotation speed of the flapping wheels on the water.

\section{The Stage of Play}

In this stage, the students have been asked to create their favorite scenes on the land or under the sea. Figure 3 shows four of these scenes, they are located respectively in the forest, in the cave, on the seamount and in a maze under the water. First, in Fig. 3(a), those trees in the forest were plant disorderly in order to exam the maneuver ability of each vehicle. Second, the chandelier cave in Fig. 3(b) indicates a very challenging environment for vehicles to pass by. Third, the seamount in Fig. 3(c)shows an uneven landscape that may cause the turnover of the vehicle with a high traveling speed.

At last, under the sea surface, a maze was constructed by one of the student groups and it provides an immersive environment to enable the students been trapped in a real maze. The existence of the maze confined the space of vehicles and may force the students to think more about the balance between the appearance and the multi-functionality. For example, the wind-powered vehicle shown in Fig. 2(c) 
cannot generate electricity under the water as in the scenes in Fig. 3(c) and Fig. 3(d).The function of the wind turbine may fail in this case. However, if we try to seek a best design of both sides, it would be helpful to modify the wind turbine with additional function that generates electricity as an ocean current turbine does under the sea.

As for fun, students can set rules of car racing game such as the overtaken is prohibited, the flying function is enabled etc. We asked students to take the snapshots for their vehicles at each level or scene so that they can share and discuss to each other on Facebook. As the advisor, we monitored the interactions of the participants by screen capturing from different viewing angles so that it can help us, as well as the students, to rethink our design of the amphibious vehicles in the later stages.
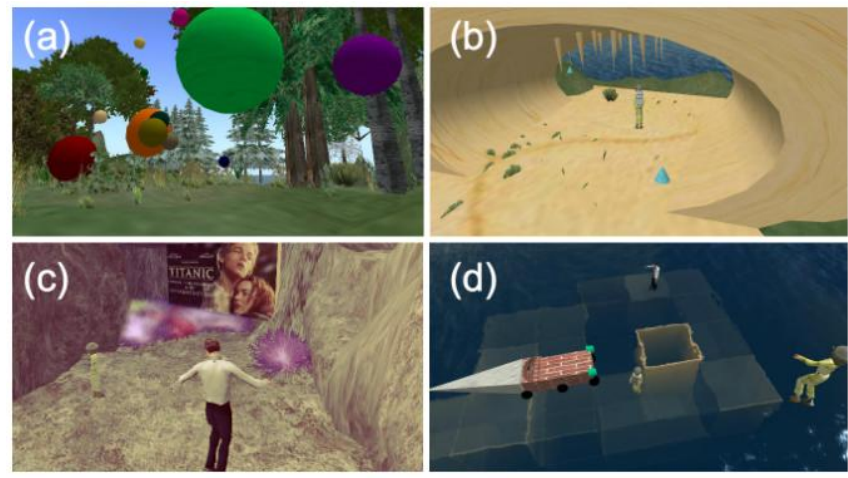

Fig. 3. Virtual scenes designed by students.

\section{The Stage of Sharing}

In this stage, all participants share their snapshots that were took in the stage of playing on the Facebook group. In these posts, the students can share their idea of design not only by the appearance of the vehicles but also the functionalities can be explained in details. We asked the students share at least three aspects for their created vehicles. For instance, in Fig. 4(a), the vehicle seems a little bit strange at the first sight because the base of the vehicle is too high that may cause the overturn of the entire vehicle. The student Miss Hong explained in her Facebook post that this vehicle is capable of driving on different landscapes such as on the sand or on the water. That is why the height of the center of mass of her vehicle is higher than those of others. In order to avoid the overturning of the vehicle, Miss Hong explained in her post saying that the relatively large wheels could solve the problem of overturning. In our opinion, the idea behind this vehicle is a good combination of a jeep and a canoe. A good jeep is designed for travelling on difficult landscapes with a high speed. And a canoe is suitable for sailing over the water alone. A combination of these two vehicles can result a competitive functionality that is suitable for her expectation. Miss Hong also explained that the windshield is two-layered in order to protect the driver from the blowing of the wind and the invading of the water.

According to the qualitative descriptions that presented on the Facebook, each vehicle has got feedbacks from the classmates as fair, good and excellent. For example, in an oval catwalk space as shown in Fig. 4(b), this vehicle has got 5 votes of excellent, 1 vote of good and 10 votes of fair. This kind of feedback is very straightforward not only from the appearance of 3D model but also the detailed explanation of the design philosophy. In this study, we encourage the students to give positive comments toward each other. This is similar to the design of the "like" button in Facebook that could lead to a gentle, positive atmosphere in the social learning process. In addition, before the students giving their comments or scores to each other, we encourage them to walk through everywhere on the oval catwalk to discover other vehicles thoroughly. Because of the $3 \mathrm{D}$ nature of this exhibition, students can explore every detail of those vehicles and then give constructive criticisms to each other. This is a genuine advantage of the social learning on the $3 \mathrm{D}$ virtual world.

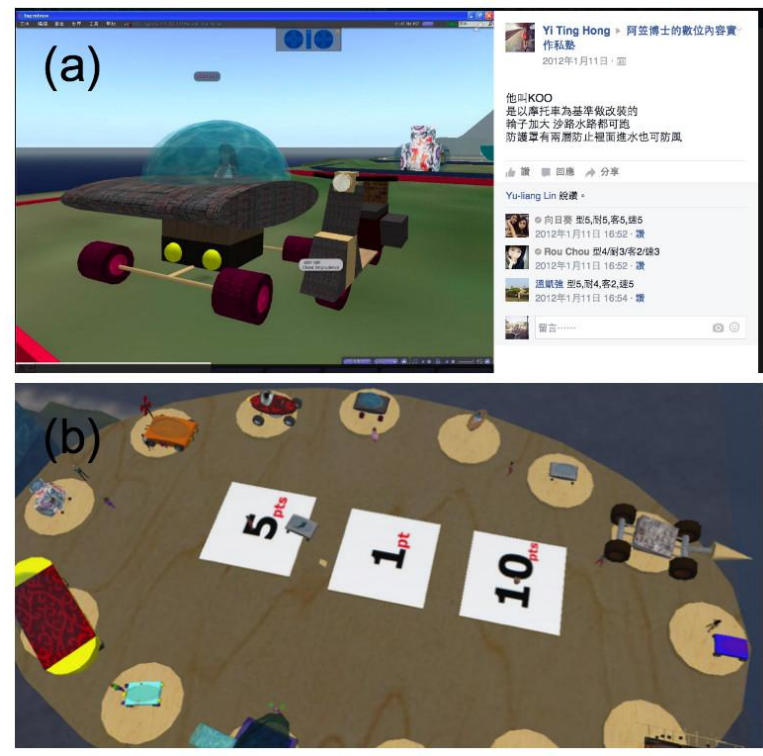

Fig. 4. Sharing the design results of amphibious vehicles (a) On the Facebook and (b) On the Opensim virtual world.

\section{E. The Stage of Reflection}

In the above stages, students have learned basic skills for constructing amphibious vehicles to satisfy their imagination, to play with the classmates in the co-create scenes and finally, to share their thoughts and experiences on the Facebook, as well as in the 3D virtual world. On the Facebook, we also asked the students to give comments with scores ranging from 1 to 5 points for four criteria: the appearance, the speed, the weather ability and the passenger capacity.

These four criteria reflect different aspects of requirement for the amphibious vehicles. First, the appearance of a vehicle represents the balance of aesthetics and mechanics. In the stage of imagination, we have introduced many successful cases that consider both aspects very well. Second, the speed of a vehicle is about the performance and is determined by the designer. Third, the weather ability is about the ability of a vehicle to survive or to withstand in different weather conditions. At last, the passenger capacity is the number of passenger that the vehicle can carry on. In short, we adopt these four criteria to a synthetic measure of the vehicle. For example, the vehicle as shown in Fig. 4(a) received an average measure as follows: 4.7 for appearance, 3.0 for speed, 3.5 for weather ability and 4.6 for passenger capacity.

After receiving peer comments according to above four 
criteria as well as some discussions in the posts, students have more information to consider their designs. In the course, we open our virtual classroom online for several weeks so that the students can $\log$ in to the virtual world at anytime they want. This strategy give them the opportunity to carefully consider the idea of amphibious vehicle and to work on what they really want to make with sufficient efforts and time.

\section{F. The Re-imagine Stage}

In this stage, the students have to re-imagine the design and to seek for possibility of better choice. It is not true that every design should be re-imagined and be modified because some vehicles are satisfactory in the first cycle of the creative thinking spiral. For those cases that are necessary to enter this stage, the designer has to think or to imagine according to more supportive materials for their design. For example, in Fig. 5(a), the student Mr. Chien-Sheng Huang has already made a lot of efforts to design his beautiful amphibious vehicle, which is inspired by a combination of motorcycle and submarine. Mr. Huang himself has great enthusiasm to make his vehicle better. We found that he took about three working days to complete his design. During these days, his design of vehicle had evolved both from the aspects of appearance and functionality to a significant leap of progress.

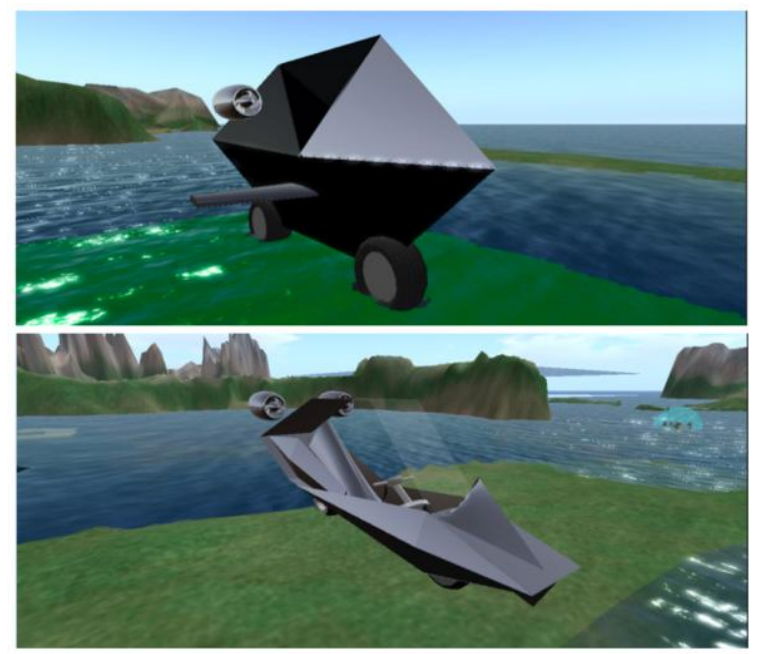

Fig. 5. The re-imagine stage of the amphibious vehicle (a) before and (b) after the modification has been made.

The original design of vehicle shown in Fig. 5(a) has a pair of side wings that provide reliable stability and superior maneuverability under the water. However, as a vehicle driving on the land, the center of gravity is too high so that it may overturn itself easily or cannot drive with high speed. In order to conquer this disadvantage, Mr. Huang tried to image more combinations of different vehicles to reach a high driving performance both in the water and on the land. Finally, in Fig. 5(b), he adopted the idea of the racing car into his original design: the side wings are removed and the body of the vehicle is reshaped to adjust the center of gravity toward a lower position so that it can keep stable in the high speed traveling. On the other hand, the upper part of the vehicle is also reshaped not only to reduce the aerodynamic drag force in a high speed but also to streamline the body of the vehicle toward a bionic mimics as a fish under the sea. The vehicle is powered by a pair of jet turbines attaching in the rear part of the vehicle.

\section{Discussions}

In this study, the students had designed the amphibious vehicles in the classroom with their classmates. Most of the time, every student can communicate with each other in many ways such as face-to-face conversations, text chats or gesture in the virtual world. These two kinds of communications are reciprocal to each other and all help to build a positive atmosphere for creative thinking. As a role of teacher or teaching assistants, we walked through every student and diagnosed their results both in a "real" classroom and a "virtual" classroom. We took snapshots of the vehicles that were built by students and gave the advices to them by walking to their seats in the real classroom. This mix-reality teaching strategy is very effective and enhances the learning process of students.

From the viewpoint of students, they had learned the skills of $3 \mathrm{D}$ modeling in the virtual world as follows. First, they learned the basic skills from the teacher via the screen casting in the classroom. Second, they explored in the virtual world and found those 3D models that were pre-implanted in the virtual world for learning. Third, the students flew back to their own islands and began to create the vehicles. During the stage of creation, we asked all the students to finish the job in silence and to prevent any disturbance to or from others.

In the fourth week, we arranged students to meet with the teacher by groups not in the time of the class in order to pre-examine the status of the design results case by case. The principle of grouping is according to their professional background. We found that the quality of design results approximately coincides with the time cost. For example, the wooden boat shown in Fig. 2(a) was built in one and a half hour that is comparatively rough than that classic car shown in Fig. 2(d) that was elaborately sculptured by two days.

\section{CONCLUDING REMARKS}

In this study, we are interested in investigating how to inspire the students to build an amphibious vehicle under the framework of creative thinking spiral in a $3 \mathrm{D}$ virtual world. Throughout the introduction in the imagine stage, the students can seek for the possibility of combining various superior functionalities into a single vehicle. In the stage of creation, the students have to construct the 3D model of amphibious vehicle according to their design idea. In this stage, the students may encounter the difficulties that they cannot build up the model realistically because of the shortage of technical background. This situation can be solved by asking others for help or by giving a detailed explanation on the post on Facebook.

To understand the quality of the results, the students designed various scenes as playgrounds for all the vehicles in the stage of playing. During the playing over different scenes, the students can learn the knowledge about the facts that if their vehicles are suitable for the scenes or not. All experiences they have learned in the stage of playing will be shared on the Facebook and look for peer comments. In this stage of sharing, the designer would introduce the idea of design and disclose the finding of the vehicle despite good or not. The peers will give qualitative and quantitative 
evaluations for each vehicle. These feedbacks become the strong supports for the students to modify or even to redesign their results in the stages of reflection and re-imagine.

\section{ACKNOWLEDGMENT}

The author would like to thank Mr. Chia-Chi Chang and Ms. L. C. Liu for their assistants of the project.

\section{REFERENCES}

[1] A. Makosz, "Using 3D virtual worlds-OpenSim, quest atlantis-to teach international school students computer science and human values," in Proc. World Conference on Educational Multimedia, Hypermedia and Telecommunications, no. 1, pp. 2480-2489, 2011.

[2] P. Baldi and C. Lopes. "The universal campus: An open 3D virtual world infrastructure for research and education," ACM eLearn Magazine, April 26, 2012.

[3] Y. -H. Chen-Burger and A. Tate, "The fish4knowledge virtual world gallery," Fish4Knowledge: Long-Term Fish Observation Using Video Processing and Visual Analytics, Springer, 2015, ch. 18, pp. 243-248.

[4] K. Hew and W. Cheung, "Use of three-dimensional (3-D) immersive virtual worlds in K-12 and higher education settings: A review of the research," British Journal of Educational Technology, vol. 41, no. 1, pp. 33-55, 2010.

[5] P. D. Bourke, "Evaluating second life for the collaborative exploration of 3D fractals," Computers \& Graphics, vol. 33, no. 1, pp. 113-117, 2009.
[6] M. Resnick, "Sowing the seeds for a more creative society," Learning and Leading with Technology, vol. 8, pp. 18-22, 2007.

[7] S. Lubar, "Was this America's first steamboat, locomotive, and car?" Invention \& Technology Magazine, vol. 21, issue 4, 2006.

[8] Simon. Rinspeedsplash hydrofoil car. [Online]. Available: http://www.simonwhatley.co.uk/rinspeed-splash-hydrofoil-car

[9] (June 15, 2005). Bionic car fueled by fishy ideas. National Geographic. [Online].

Available: http://news.nationalgeographic.com/news/2005/06/0615_050615_fis hcar.html

[10] M. Hanlon, "The bionic car project," Gizmag, December 2015.

[11] O. Nancy, "EDAG car with textile skin set for Geneva show," Tech Xplore, December 2014.

[12] S. Kiattisak, "Floating bike for Thai flooding situation," YouTube, Oct. 2011.

[13] H. K. Kaspar, "Homemade JETSKI using a motorbike saves us from the Bangkok floods," YouTube, Dec., 2011

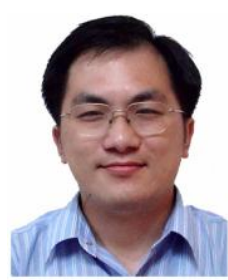

Hung-Cheng Chen received the B.E. in harbor and river engineering from National Taiwan Ocean University, Taiwan, in 1994. He received his M.S. in agricultural engineering and his Ph.D. in applied mechanics from National Taiwan University, Taiwan, in 1996 and 2001, respectively. He is now an assistant professor of the Faculty of the Center of General Education, Yu Da University of Science and Technology. His research interests include educational technology, digital art and sustainable tourism. 